\title{
Presentación. Tecnologías de mejora humana, debate ético e impacto sociocultural
}

Human enhancement technologies: ethical debate and sociocultural impact

Miguel Moreno Muñoz

Profesor Contratado Doctor (acreditado para TU). Departamento de Filosofía II, Universidad de Granada. España mm3@ugr.es

Francisco Lara Sánchez

Profesor Titular de Universidad. Departamento de Filosofía I, Universidad de Granada. España flara@ugr.es

\author{
TECNOLOGÍAS DE MEJORA HUMANA: DEBATE ÉTICO E IMPACTO SOCIOCULTURAL \\ MONOGRÁFICO COORDINADO POR MIGUEL MORENO (Universidad de Granada) y FRANCISCO LARA (Universidad de Granada)
}

\section{RESUMEN}

Los artículos de este volumen analizan el impacto socio-cultural de diversas tecnologías de mejora cognitiva y funcional aplicables en seres humanos. Sus autores aportan elementos teóricos, criterios metodológicos y enfoques especializados sobre aspectos sujetos a un intenso debate en la literatura reciente. En conjunto, contribuyen a contextualizar de modo riguroso una problemática compleja, cuyo carácter interdisciplinar y rápida evolución plantea dificultades específicas para las ciencias sociales.

\section{ABSTRACT}

The papers in this issue analyze the socio-cultural impact of diverse cognitive and functional enhancement technologies applicable in humans. The authors provide theoretical or methodological elements, and specialized approaches on topics under discussion in the recent literature. All of them contribute to contextualize rigorously a complex set of problems, particularly challenging for social sciences because of their interdisciplinary nature and rapid evolution.

\section{PALABRAS CLAVE}

tecnologías de mejora humana | bioética | mejora cognitiva | mejora funcional | mejora genética KEYWORDS

human enhancement technologies | bioethics | cognitive enhancement | functional enhancement | genetic enhancement

El desarrollo y la convergencia de tecnologías susceptibles de ser aplicadas con objetivos de mejora de las capacidades humanas ha generado en los últimos quince años una ingente bibliografía. El debate académico ha sido intenso, centrado inicialmente en los aspectos controvertidos de posibles aplicaciones biotecnológicas que hoy resultan combinables con desarrollos cada vez menos especulativos en nanotecnología, robótica avanzada y neuromodulación, entre otros.

Considerando la velocidad a la que se sucenden avances en dominios diversos de las ciencias biomédicas (genética, neurociencias, bioingeniería, etc.) muchos autores piensan que no está lejos el día en que será posible emplear la biotecnología para algo más que prevenir o tratar enfermedades. Por ejemplo, para mejorar numerosos aspectos de la naturaleza y la existencia humana mediante la modulación de rasgos de propósito general implicados en la inteligencia, la memoria, la respuesta emocional y la percepción de bienestar.

El empleo de las biotecnologías de mejora para extender el horizonte de longevidad, salud y calidad de vida hasta límites que hoy resultan poco verosímiles puede redefinir los patrones dominantes de felicidad y sustentar concepciones radicalmente nuevas sobre las posibilidades asociadas con una vida humana plena y fructífera. Pero también pueden cuestionar nociones antropológicas fundamentales: desafían los modos establecidos de construir la identidad individual y de entender nuestro potencial como seres humanos; distorsionan las nociones que sustentan el sentido de la dignidad humana y sus concreciones normativas; y dificultan la percepción de valor que asociamos con ciertos rasgos de la sociedad o de la naturaleza humana y los fines de la medicina.

Las contribuciones recogidas en este volumen monográfico de Gazeta de Antropología se han solicitado a investigadores con una especialización acreditada en la reflexión bioética y abordan aspectos sustantivos del debate, todos ellos novedosos e interesantes a juicio de los coordinadores (1). 
Entre los artículos dedicados a la mejora cognitiva, el de Paloma García Díaz se centra en la expectación pública y el debate bioético especializado que ha generado el posible uso de nootrópicos para aumentar nuestras potencialidades cognitivas. Estas sustancias podrían mejorar la memoria, la atención, el aprendizaje y los procesos de toma de decisiones. Por ello, la investigación clínica en estos productos parece responder a una necesidad social. Disponer en el mercado de sustancias que nos permitan un mayor rendimiento intelectual concierne particularmente a sectores como profesionales de la sanidad, investigadores, estudiantes y personal militar, entre otros. El modafinilo (@Provigil) juega un papel destacado en el conjunto de los fármacos de neuromejora, pues se considera como la primera sustancia que merece en sentido estricto el calificativo de nootrópico. Asimismo, el modafinilo parece presentar menos contraindicaciones que otras sustancias como el metilfenidato (Ritalin@) o las sales mixtas de anfetaminas (@Adderall).

Sobre mejora cognitiva trata también la contribución de Miguel Moreno Muñoz. El autor señala las oportunidades para ciertos programas de mejora cognitiva no convencional abiertas por los desarrollos tecnológicos ligados al núcleo de innovaciones características de la cuarta revolución industrial. Tras analizar la evolución de los programas de automatización del trabajo, el autor propone un ejercicio de prospectiva sustentado en la literatura reciente sobre el desempleo estructural como fenómeno y tendencia característica de las sociedades contemporáneas. Como hipótesis de trabajo asume que esta tendencia puede resultar reforzada por la implantación de las tecnologías asociadas con la cuarta revolución industrial. Pero se distancia de los enfoques habituales en la importancia que otorga a ciertos factores para entender el desfase progresivo entre desarrollo tecnológico y las dinámicas de adaptación/transformación de los sistemas educativos durante la segunda mitad del siglo XX. Entre otros, el autor señala los efectos de la digitalización y la incorporación de inteligencia artificial en el desarrollo de sistemas robóticos, cuyas capacidades compiten ya o sobrepasan a las de trabajadores especializados en tareas complejas. Su trabajo concluye con la propuesta de un programa de mejora cognitiva no convencional que considera no solo viable en el contexto actual de posibilidades científicotecnológicas, sino recomendable para compensar la evolución constatada de los indicadores de eficiencia de los sistemas educativos públicos y privados durante la última década.

Conectando la mejora cognitiva y funcional, José Luis Pérez Triviño examina las valiosas implicaciones que para el deporte conllevaría el mejoramiento de las capacidades cognitivas, dada la innegable relevancia de estas para el rendimiento físico. Se aborda el impacto de la neurociencia en el deporte y, en particular, el uso de dos técnicas: los mejoradores cognitivos y los estimuladores transcraneales. Se señalan sus efectos y sus inconvenientes, en especial, en la práctica deportiva. Dada la relativa novedad de la estas técnicas y sustancias mejoradoras, y la ausencia de pruebas concluyentes de sus efectos a corto y largo plazo, el autor defiende que su uso debe estar regido por un escrupuloso respeto del principio de precaución.

Pero las capacidades humanas que podrían ser mejoradas actualmente, dados los últimos avances en biotecnología, no se reducen a las cognitivas y físicas. También están las relacionadas con la motivación y la deliberación moral. En este terreno, el artículo de Francisco Lara pretende provocar un debate sobre la racionalidad o no de usar determinadas sustancias para mejorar el comportamiento moral de los seres humanos. Se recogen las posibilidades al respecto de sustancias como la serotonina y la oxitocina, y se presenta la pregunta de si, en el caso de no conllevar efectos secundarios no deseados, su uso para la mejora moral no podría resultar coherente con la indiscutible pretensión de combatir por medios educativos o jurídicos las negativas consecuencias personales y sociales del comportamiento inmoral. Esta posible biomejora moral podría presentarse, además, como ineludible si nos fijamos en las dificultades de los seres humanos para afrontar con sus intuiciones morales las graves amenazas a su supervivencia que conllevan un entorno globalizado muy distinto al que provocó evolutivamente esas intuiciones. En esta contribución el autor también analiza la objeción de que intervenir biológicamente a los seres humanos para mejorar su comportamiento moral podría mermar la autonomía e integridad personal de los seres mejorados.

Pablo García Barranquero pretende familiarizar al lector con algunas de las cuestiones más interesantes que plantean los programas de investigación para contrarrestar los procesos de envejecimiento y extender de modo radical la vida humana. En su artículo se analizan los puntos esenciales de la atrevida teoría de Aubrey de Grey sobre los últimos avances en gerontología: concepto de envejecimiento, evolución de la esperanza de vida en el siglo XX (ligado al concepto de vida máxima), la guerra contra el envejecimiento o la velocidad de escape en longevidad. Esto le permite conectar y reflexionar brevemente sobre dos temas capitales en la filosofía actual, desde una perspectiva algo más humanista: el mejoramiento humano y la idea de inmortalidad.

En la contribución de Javier Rodríguez Alcázar confluyen dos ámbitos clave de debate en la filosofía 
práctica contemporánea. Uno es la discusión en torno a la prelación entre la ética y la política, con una derivada metafilosófica relativa a las relaciones entre filosofía moral y filosofía política. Estas cuestiones han recorrido toda la tradición filosófica occidental y han recibido un nuevo impulso con la publicación del libro póstumo de Bernard Williams In the Beginning was the Dead: Realism and Moralism in Political Argument. El segundo ámbito es la discusión en ética aplicada acerca de las implicaciones morales de las tecnologías de mejora humana. El artículo pretende iluminar cada uno de esos ámbitos desde el otro. Por una parte, denuncia que la reflexión sobre las tecnologías de mejora ha incorporado de forma muy deficiente los aspectos políticos de dichas tecnologías y adolece de los defectos del moralismo político. Por otra, encuentra en la reflexión filosófica sobre la mejora humana un arsenal de ejemplos que permiten desacreditar ese moralismo político sin caer en los excesos del realismo político, otorgando así crédito a la posición defendida por el autor, el minimalismo político.

Este volumen cuenta con la contribución desinteresada del profesor Julian Savulescu, un experto de referencia en la literatura internacional sobre la problemática (2). El autor analiza las principales objeciones de los autores que se han mostrado críticos con el uso de tecnologías para modificar o mejorar a los seres humanos. Centra sus argumentos en un tipo de intervenciones controvertidas, como son las dirigidas a modificar ciertos rasgos de la dotación genética bajo determinados supuestos. Sin minusvalorar las objeciones, Savulescu analiza diversos contextos en los que las modificaciones biológicas pueden mejorar las oportunidades de un individuo humano y favorecer así una vida mejor. En tales casos, sostiene que tenemos razones poderosas para permitir la modificación de rasgos biológicos propios o de la descendencia, y carecería de justificación no hacerlo. El interés de su trabajo estriba en mostrar la continuidad existente entre ciertos tipos de mejora convencionales (mediante suplementos dietéticos o modificaciones ambientales) y las posibilidades abiertas por intervenciones biotecnológicas más radicales. El autor enfatiza la continuidad de objetivos entre los programas para prevenir o curar enfermedades y otros programas de mejora, puesto que atribuye un valor instrumental a la salud, como medio para desarrollar una vida mejor. Además de prevenir enfermedades, las tecnologías disponibles pueden contribuir a ampliar las oportunidades de muchos individuos para aspirar a una vida mejor. Facilitar el acceso a las biotecnologías de mejora sería un modo de concretar el criterio de beneficencia.

\section{Notas}

1. El trabajo ha contado con el apoyo del proyecto FFI2012-32565: Ética y política de los avances biomédicos en mejora humana. Convocatoria 2012, subprograma de Proyectos de investigación fundamental no orientada.

2. Los coordinadores agradecen a Oxford University Press la autorización para reproducir en este monográfico la contribución del profesor Savulescu. 